\title{
Tanshinone IIA blocks epithelial-mesenchymal transition through HIF-1 $\alpha$ downregulation, reversing hypoxia-induced chemotherapy resistance in breast cancer cell lines
}

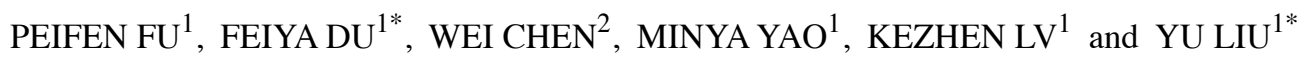 \\ ${ }^{1}$ Department of Breast Surgery Center, The First Affiliated Hospital, ${ }^{2}$ Department of Hepatobiliary and Pancreatic Surgery, \\ Second Affiliated Hospital, School of Medicine, Zhejiang University, Hangzhou, Zhejiang 310003, P.R. China
}

Received February 14, 2014; Accepted March 19, 2014

DOI: $10.3892 /$ or.2014.3140

\begin{abstract}
The aim of the present study was to investigate the effects of tanshinone IIA (Tan IIA), an active constituent of Salvia miltiorrhiza Bunge, on epithelial-mesenchymal transition (EMT) and hypoxia-induced chemoresistance in breast cancer cells. To induce hypoxia, MCF-7 and HCC1973 cells were treated with $100 \mu \mathrm{M}$ deferoxamine followed by doxorubicin (DOX). Cell viability and proliferation were examined using the CCK-8 and EdU assays, respectively. Western blot and immunofluorescence analyses of the expression of two EMT markers, E-cadherin and vimentin, were also carried out. The role of HIF-1 $\alpha$ and TWIST in mediating the effects of Tan IIA was determined through siRNA. Based on the results, hypoxia-induced DOX resistance was observed in both MCF-7 and HCC1973 cells (both $\mathrm{P}=0.001$ ), which was reversed with Tan IIA. Specifically, in hypoxic conditions, Tan IIA significantly decreased cell viability and proliferation (all $\mathrm{P} \leq 0.001$ ), but not apoptosis. Hypoxia also significantly reduced $E$-cadherin and increased vimentin protein levels $(\mathrm{P} \leq 0.005)$, which returned to control levels with Tan IIA. In addition, silencing both HIF-1 $\alpha$ and TWIST expression abrogated the effects of Tan IIA on cell viability. Taken together, Tan IIA ameliorated hypoxia-induced DOX resistance and EMT in breast cancer cell lines, which may be attributed to the downregulation of HIF-1 $\alpha$ expression. Further in vivo studies, however, are required to fully elucidate the therapeutic potential of Tan IIA in increasing the sensitivity of breast cancer cells to chemotherapy.
\end{abstract}

Correspondence to: Dr Peifen Fu, Department of Breast Surgery Center, The First Affiliated Hospital, School of Medicine, Zhejiang University, 79 Qingchun Road, Hangzhou, Zhejiang 310003, P.R. China E-mail: fupeifen@hotmail.com

\section{${ }^{*}$ Contributed equally}

Key words: breast cancer, chemotherapy resistance, HIF-1 $\alpha$, hypoxia, tanshinone IIA

\section{Introduction}

Despite advancements in tumor screening and detection as well as development of new treatments, breast cancer remains the leading cause of cancer-related mortality for women worldwide (1). In solid tumors, certain regions may become hypoxic (2); however, tumor cells overcome this condition through increased angiogenesis, glycolysis, growth factor expression as well as inhibition of apoptosis (3). In some cases, hypoxia can induce resistance to radiotherapy and chemotherapy and increase metastasis (4) due, in part, to the downregulation of adhesion molecules (5).

Certain genes are altered in the presence of hypoxia, including hypoxia-inducible factor 1 (HIF-1) (4), a heterodimer consisting of HIF- $1 \alpha$ and HIF-1 $\beta$ transcription factors (6). In contrast to the constitutively expressed nuclear HIF-1 $\beta$ (ARNT) (7), HIF-1 $\alpha$ is a cytoplasmic protein that is upregulated in response to hypoxia. In normoxia, HIF-1 $\alpha$ is hydroxylated via $\mathrm{O}_{2}$-dependent enzyme activity, resulting in ubiquitin-proteasome-mediated degradation (8). Hypoxiainduced radioresistance of some tumor cells is mediated by HIF-1 (9). Moreover, a role for HIF in epithelial-mesenchymal transition (EMT) and prostate cancer cell migration has been reported (10).

Tanshinone IIA (Tan IIA), a major lipophilic component found in Salvia miltiorrhiza Bunge root extract, has been used to treat myocardial infarction, angina pectoris, stroke, diabetes, and sepsis (11). In addition, Tan IIA alleviated residual tumor hypoxia and inhibited EMT in vivo without altering HIF-1 $\alpha$ expression (12). Thus, the present study examined the hypothesis that Tan IIA downregulates HIF-1 $\alpha$ and blocks EMT, thereby reversing hypoxia-induced chemoresistance in breast cancer cells.

\section{Materials and methods}

Cell culture, induction of hypoxia and Tan IIA treatment. MCF-7 cells were maintained in Dulbecco's modified Eagle's medium (DMEM) containing 10\% fetal bovine serum (FBS) and $1 \%$ penicillin/streptomycin at $37^{\circ} \mathrm{C}$ in an environment with $5 \% \mathrm{CO}_{2}$. All culture reagents were purchased from Life Technologies (Carlsbad, CA, USA). HCC1937 cells were 
maintained in RPMI-1640 medium containing 10\% FBS and $1 \%$ penicillin/streptomycin at $37^{\circ} \mathrm{C}$ in an environment with $5 \% \mathrm{CO}_{2}$. To induce hypoxia, cells were treated with $100 \mu \mathrm{M}$ deferoxamine (Novartis, Basel, Switzerland) for $24 \mathrm{~h}$ as previously reported (13). Cells in the Tan IIA groups received $10 \mu \mathrm{M}$ Tan IIA (Nanjing Zelang Medical Technology Co., China).

CCK-8 assay. MCF-7 cells were maintained in MEM containing $10 \%$ FBS and seeded onto 96-well plates at a density of $1 \times 10^{4}$ cells/well. HCC1937 cells were grown in RPMI-1640 containing 10\% FBS and seeded onto 96-well plates at a density of $5 \times 10^{3}$ cells/well. On the next day, cells were cultured in serum containing antibiotic-free medium with $100 \mu \mathrm{M}$ deferoxamine with and without $10 \mu \mathrm{M}$ Tan IIA for $48 \mathrm{~h}$ at $37^{\circ} \mathrm{C}$ after which $100 \mu 1$ of CCK-8 solution (Dojindo, Kumamoto, Japan) was added per well for an additional $3 \mathrm{~h}$ at $37^{\circ} \mathrm{C}$. The optical density (OD) was measured at $450 \mathrm{~nm}$ with an MRX II microplate reader (Dynex, Chantilly, VA, USA).

Transfection of HIF-1 $\alpha$ and TWIST siRNA. Scrambled, HIF-1 $\alpha$ and TWIST siRNA were purchased from Santa Cruz Biotechnology (Santa Cruz, CA, USA). siRNAs (100 nM) were transfected into cells in the presence of Lipofectamine 2000 transfection reagent (Invitrogen, Carlsbad, CA, USA) following the manufacturer's instructions. After 6-8 h, the medium was removed and cells were maintained in normal medium for an additional $24 \mathrm{~h}$.

Western blot assay. Cells were washed with cold PBS and treated with lysis buffer (Cell Signaling, Danvers, MA, USA) at $4^{\circ} \mathrm{C}$ or on ice for $2 \mathrm{~h}$. After the protein concentration was determined with BCA kit (Thermo Fisher Scientific, Rockford, IL, USA), proteins ( $40 \mu \mathrm{g}$ ) were separated by SDS-PAGE and transferred onto a PVDF membrane (Millipore, Billerica, MA, USA). After the membranes were blocked with $5 \%$ bovine serum albumin (BSA) in $0.1 \%$ Tween-20 (TBS/T) on ice for $2 \mathrm{~h}$, they were incubated with the following primary antibodies (1:1,000; all from Abcam, Cambridge, MA, USA) at $4^{\circ} \mathrm{C}$ overnight: HIF- $1 \alpha$, E-cadherin, vimentin and $\beta$-actin. The membranes were then incubated with the appropriate secondary antibody (1:2,000; Abcam) at room temperature for $2 \mathrm{~h}$. Bands were visualized by chemiluminescence (GE Healthcare, Piscataway, NJ, USA), and the membranes were exposed to film (Kodak, Rochester, NY, USA).

Immunofluorescence staining. After the cells were washed with cold PBS and fixed in 4\% paraformaldehyde for $15 \mathrm{~min}$, they were blocked with 5\% BSA at room temperature for $30 \mathrm{~min}$. The cells were next incubated with anti-E-cadherin or anti-vimentin antibodies (1:200; Abcam) at $4^{\circ} \mathrm{C}$ overnight. After washing with PBS, cells were treated with FITC- or CY3-conjugated secondary antibodies (1:200; Abcam) at room temperature for $2 \mathrm{~h}$. Nuclear staining was performed with DAPI (Sigma) at room temperature for $2 \mathrm{~min}$. Following washing in PBS twice, observation was performed under an inverted fluorescence microscope (Olympus, Tokyo, Japan).

Flow cytometry. The BU-Annexin V-FITC apoptosis detection kit (Biouniquer Technology Co., Nanjing, China) was used to evaluate the effects of DOX and Tan IIA on apoptosis. Cells were digested with an EDTA-free trypsin solution $(0.25 \%$; Life Technologies), and a single-cell suspension $\left(1-5 \times 10^{6}\right)$ was prepared. After washing in PBS twice, $100 \mu \mathrm{l}$ of Binding Buffer and $5 \mu \mathrm{l}$ of FITC-conjugated Annexin-V $(20 \mu \mathrm{g} / \mathrm{ml})$ were added and incubated at room temperature in the dark for $30 \mathrm{~min}$. Following addition of $5 \mu \mathrm{l}$ of propidium iodide (PI) at $50 \mu \mathrm{g} / \mathrm{ml}$ for $5 \mathrm{~min}$ in the dark, $400 \mu \mathrm{l}$ of Binding Buffer was added. Flow cytometry was performed immediately (within $1 \mathrm{~h}$ ) with a FACScan flow cytometer (BD Biosciences, San Jose, CA, USA). In the negative control groups, Annexin V-FITC and/or PI were not added.

5-Ethynyl-2'-deoxyuridine (EdU) assay. MCF-7 and HCC1937 were seeded onto 96 -well plates at a density of $3 \times 10^{3}$ cells/well in their respective growth media. The medium was replaced with the corresponding serum-free medium to synchronize the cells. After $24 \mathrm{~h}$, the serum-free medium was replaced with growth media containing $100 \mu \mathrm{M}$ deferoxamine to induce hypoxia as well as $0.2 \mu \mathrm{g} / \mathrm{ml}$ DOX without and with $10 \mu \mathrm{M}$ Tan IIA for $48 \mathrm{~h}$. Cell proliferation was assessed using an EdU assay using the Click-iTEdU imaging kit (Invitrogen) according to the manufacturer's instructions.

Statistical analysis. Data are expressed by the mean and standard deviation (SD). Comparisons among two independent groups were performed by the two independent samples t-test; comparisons among three or more independent groups were performed by the one-way ANOVA with the Bonferroni post-hoc test. The relative cell viabilities between various treatment groups and various DOX dosages were evaluated by ANCOVA with one covariate of DOX dosage. The twotailed P-values $<0.05$ were considered to indicate statistically significant differences. Statistical analyses were performed using SPSS 15.0 (SPSS, Chicago, IL, USA).

\section{Results}

Hypoxia induces resistance to DOX, which is reversed with Tan IIA. To investigate the sensitivity of MCF-7 and HCC1937 cells to DOX in normoxia and hypoxia, a CCK-8 assay was employed. For both MCF-1 and HCC1937 cells, the tumor cell viabilities were significantly decreased with the increasing DOX concentrations $(\mathrm{P}<0.001$; Fig. $1 \mathrm{~A}$ and $\mathrm{B})$. In the presence of $0.25,0.5$, and $1 \mu \mathrm{g} / \mathrm{ml}$ a significantly higher cell viability was observed when cells were cultured in hypoxic conditions as compared to normoxia ( $\mathrm{P}=0.001$; Fig. $1 \mathrm{~A}$ and $\mathrm{B})$. Thus, we confirmed that hypoxia induced DOX resistance in MCF-1 and HCC1937 cancer cell lines.

To determine if Tan IIA could reverse the hypoxia-induced DOX resistance, cells were next cultured in hypoxic conditions without and with Tan IIA. As shown in Fig. 1C, the relative cell viability of MCF-7 cells cultured in hypoxia and treated with DOX+Tan IIA was significantly decreased as compared to the cells treated with DOX for $24 \mathrm{~h}$ (50.7 vs. $72.3 \%$, respectively, $\mathrm{P}<0.001), 48 \mathrm{~h}(46.1$ vs. $75.9 \%$, respectively, $\mathrm{P}<0.001)$, and $72 \mathrm{~h}$ (35.7 vs. $63.7 \%$, respectively, $\mathrm{P}<0.001$ ). Similar results were also observed in HCC1937 cells (all time points, $\mathrm{P}<0.001$; Fig. 1D), suggesting that Tan IIA may reduce the resistance to DOX induced by hypoxia. 

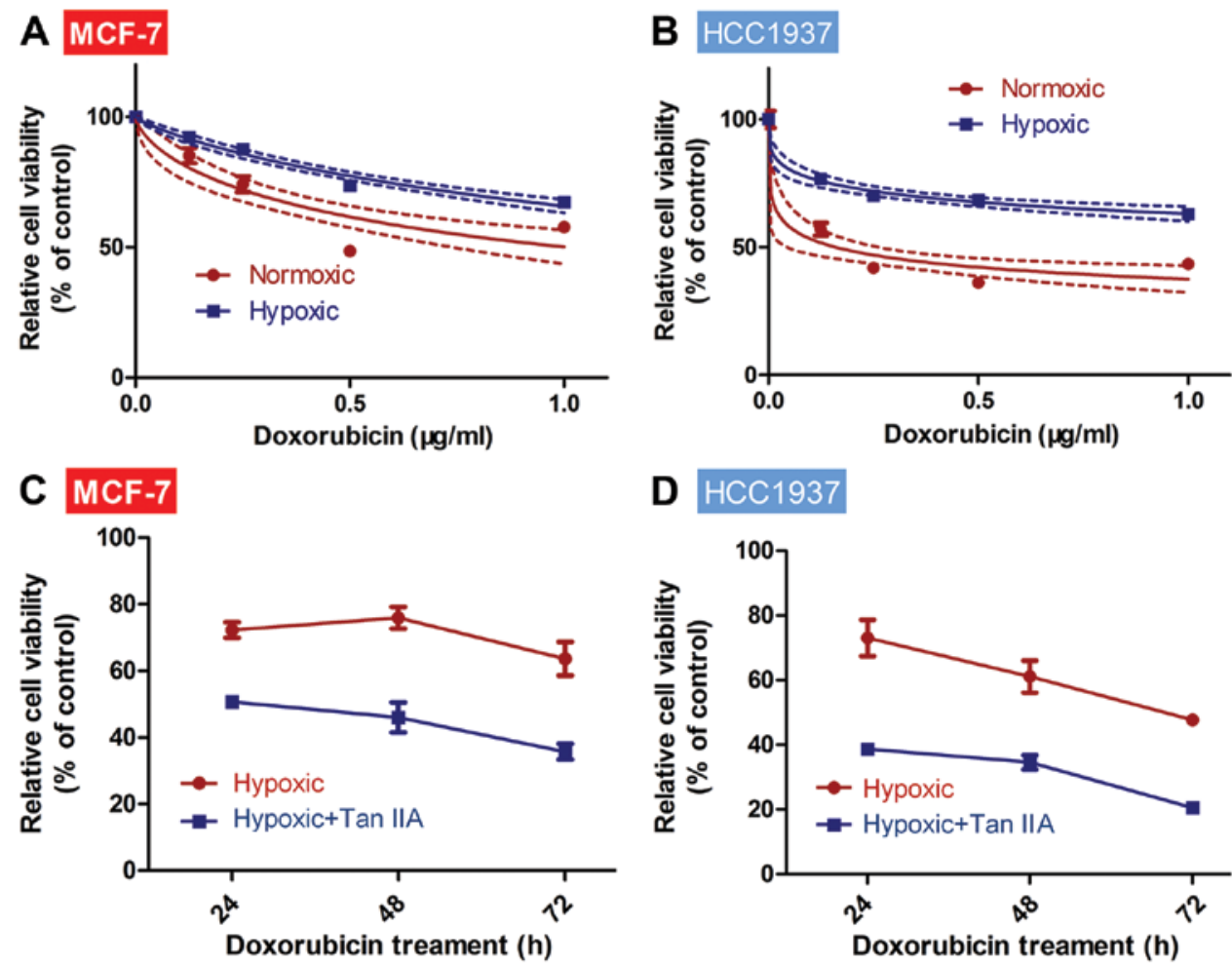

E MCF-7
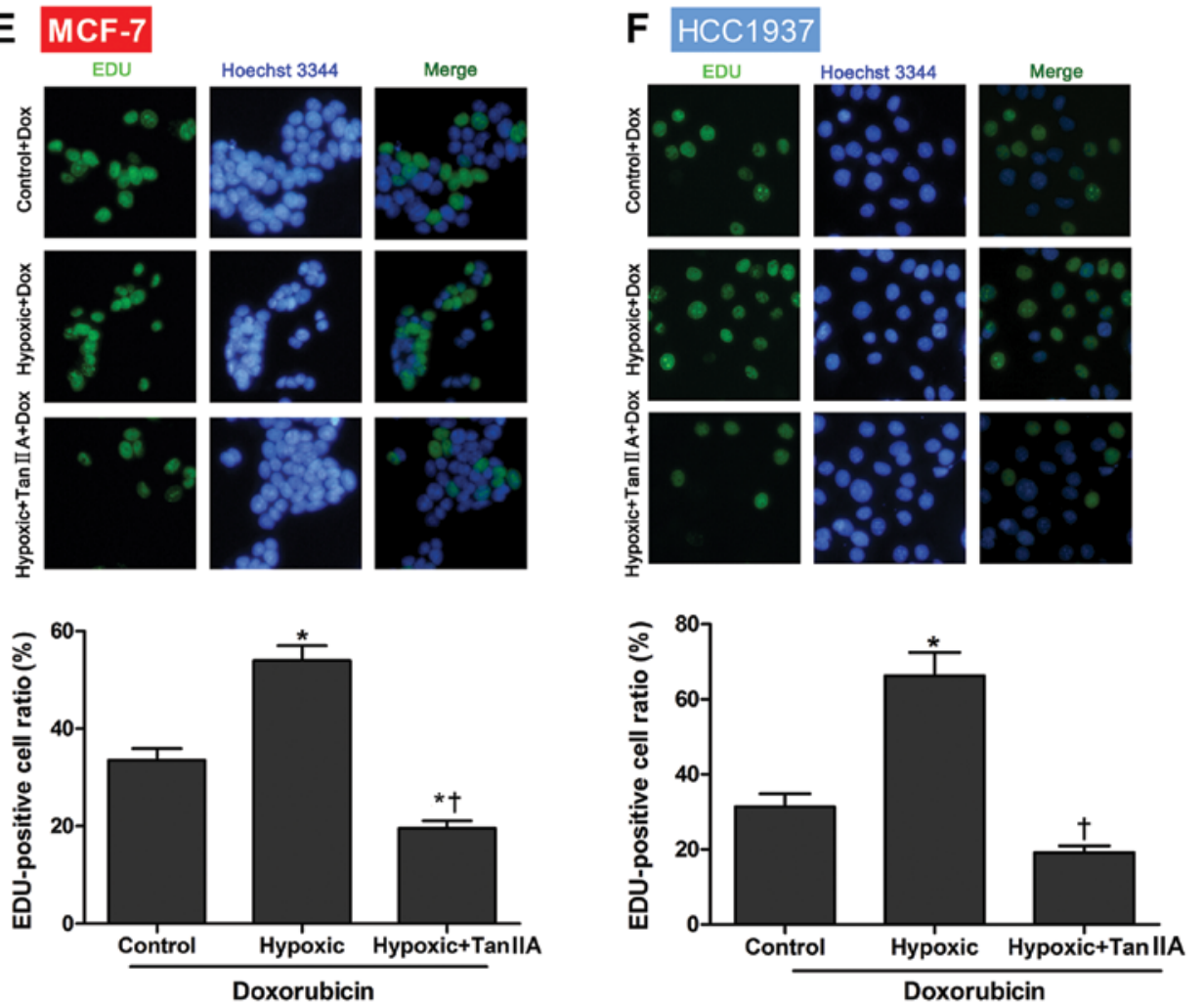

Figure 1. Tan IIA reverses hypoxia-induced DOX resistance in breast cancer cell lines. (A-D) Cell viability and (E and F) proliferation of MCF-7 and HCC1937 cells was determined using the CCK-8 and EdU assays. (A) MCF-7 and (B) HCC1937 cell viability in hypoxic (100 $\mu$ M deferoxamine) and normoxic conditions with the indicated DOX dosages (relative to control) for 24 h. (C) MCF-7 and (D) HCC1937 cell viability in hypoxic conditions after treatment with DOX $(0.2 \mu \mathrm{g} / \mathrm{ml})$ and DOX+Tan IIA $(10 \mu \mathrm{M})$ for the indicated time points (relative to control). (E) MCF-7 and (F) HCC1937 cell proliferation in the control+DOX, hypoxia+DOX, and hypoxia+DOX+Tan IIA treatment groups. " $\mathrm{P}<0.05$ compared to the control group; ${ }^{\dagger} \mathrm{P}<0.05$ compared to the hypoxia group.

Effects of hypoxia, DOX and Tan IIA on cell proliferation. The effects of hypoxia and Tan IIA on cell proliferation were next assessed in the presence of $0.2 \mu \mathrm{g} / \mathrm{ml}$ DOX using the
EdU assay. As shown in Fig. 1E and F, culturing either MCF-7 or HCC1937 cells in hypoxia significantly increased the proportion of EdU-positive cells as compared to the control 
A MCF-7
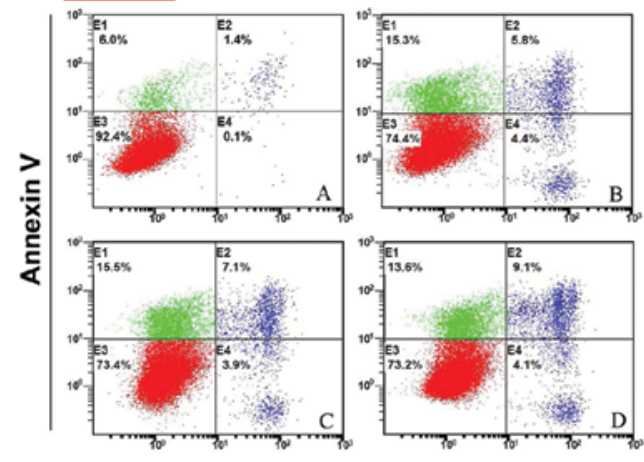

PI

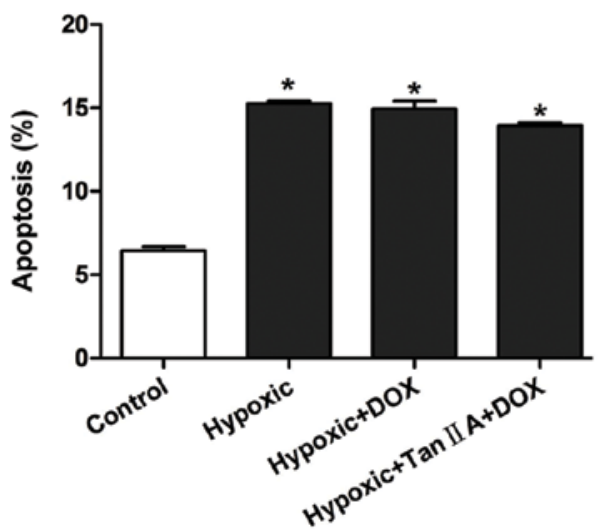

B $\mathrm{HCC1937}$

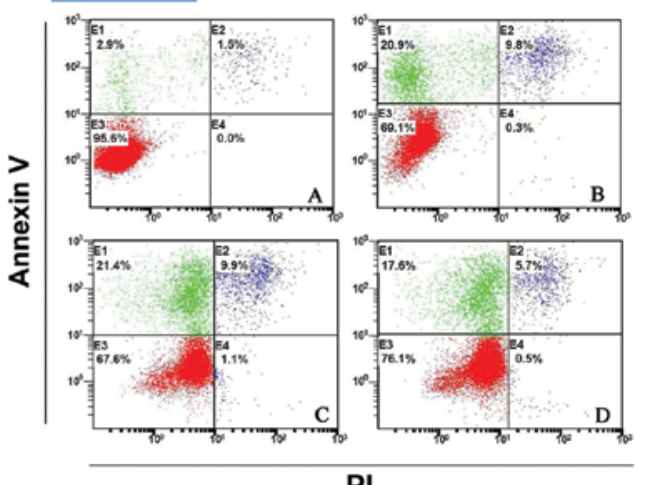

PI

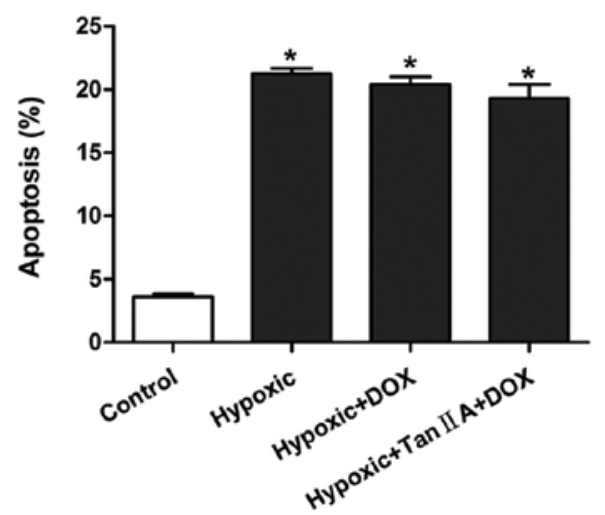

Figure 2. Tan IIA does not induce (A) MCF-7 or (B) HCC1937 cell apoptosis. Apoptosis was determined using flow cytometry in the following treatment groups: control (normoxia), hypoxia, hypoxia+DOX $(0.2 \mu \mathrm{g} / \mathrm{ml})$, hypoxia+DOX+Tan IIA $(10 \mu \mathrm{M})$. ${ }^{*} \mathrm{P}<0.05$ compared to the control group.

(normoxia, both $\mathrm{P}=0.001$ ). Furthermore, cell proliferation was significantly reduced in the presence of hypoxia with the addition of Tan IIA (both $\mathrm{P}<0.001$ ).

Effects of hypoxia, DOX and Tan IIA on cell apoptosis. To determine if Tan IIA increased the sensitivity of MCF-7 or HCC1937 cells to DOX by inducing apoptosis, flow cytometry was performed to measure apoptosis rates in the presence of hypoxia, DOX, and Tan IIA. As shown in Fig. 2A and B, the apoptosis rates of both MCF-1 and HCC1937 cells were significantly higher than control when cultured in the presence of hypoxia, hypoxia+DOX, or hypoxia+DOX+Tan IIA (all $\mathrm{P}<0.001)$. However, no significant differences were observed between the three groups, indicating that the reduced viability observed with Tan IIA was not due to apoptosis induction.

Effects of hypoxia, DOX and Tan IIA on EMT. To determine if the effects of hypoxia and Tan IIA were mediated by changes in EMT, E-cadherin and vimentin protein expression was determined by western blot analysis. As shown in Fig. 3A and B, E-cadherin protein expression was significantly decreased in response to hypoxia (both $\mathrm{P}<0.001$ ). Treatment with Tan IIA significantly increased it, but not to control levels in MCF-7 cells (both $\mathrm{P}=0.002$ ). In contrast, vimentin expression levels were significantly increased in the hypoxia group compared to control in both cell lines $(\mathrm{P} \leq 0.002)$. Treatment with Tan IIA ameliorated the effects of hypoxia on vimentin expression (both $\mathrm{P} \leq 0.005$; Fig. $3 \mathrm{~A}$ and $\mathrm{B}$ ). Similar results were observed with immunofluorescence analysis (Fig. 3C).

Given the importance of TWIST regulation by HIF- $1 \alpha$ in EMT (14), the effects of its knockdown were next assessed in cells cultured in the presence of hypoxia and Tan IIA. As shown in Fig. 4A, TWIST siRNA reduced TWIST protein expression in both MCF-1 and HCC1937 cells. After TWIST knockdown, no significant difference in tumor cell viability was observed between the hypoxia and hypoxia+Tan IIA groups in response to DOX (Fig. 4B and C). These results suggest that Tan IIA may inhibit hypoxia-induced EMT.

Effects of Tan IIA on cell viability and proliferation are mediated by HIF-1 $\alpha$ expression. As shown in Fig. 3A and B, $\mathrm{HIF}-1 \alpha$ expression levels were significantly increased in the hypoxia group compared to control in both cell lines (both $\mathrm{P}<0.001$ ), and treatment with Tan IIA ameliorated the effects of hypoxia on HIF-1 $\alpha$ expression (both $\mathrm{P} \leq 0.005$ ).

To determine if the effects of Tan IIA were mediated by HIF-1 $\alpha$, both MCF-1 and HCC1937 cells were transfected with HIF-1 $\alpha$ siRNA. As shown in Fig. 5A, transfection of both MCF-1 and HCC1937 cells with HIF-1 $\alpha$ siRNA reduced HIF-1 $\alpha$ protein expression levels. In HIF-1 $\alpha$ siRNA-transfected cells, no significant differences in cell viability (Fig. 5B and C) and proliferation (Fig. 5D and E) in response to DOX were observed between the hypoxia and hypoxia+Tan IIA groups. These results suggest that HIF-1 $\alpha$ mediates the biological effects of Tan IIA. 
A $\mathrm{MCF}-7$
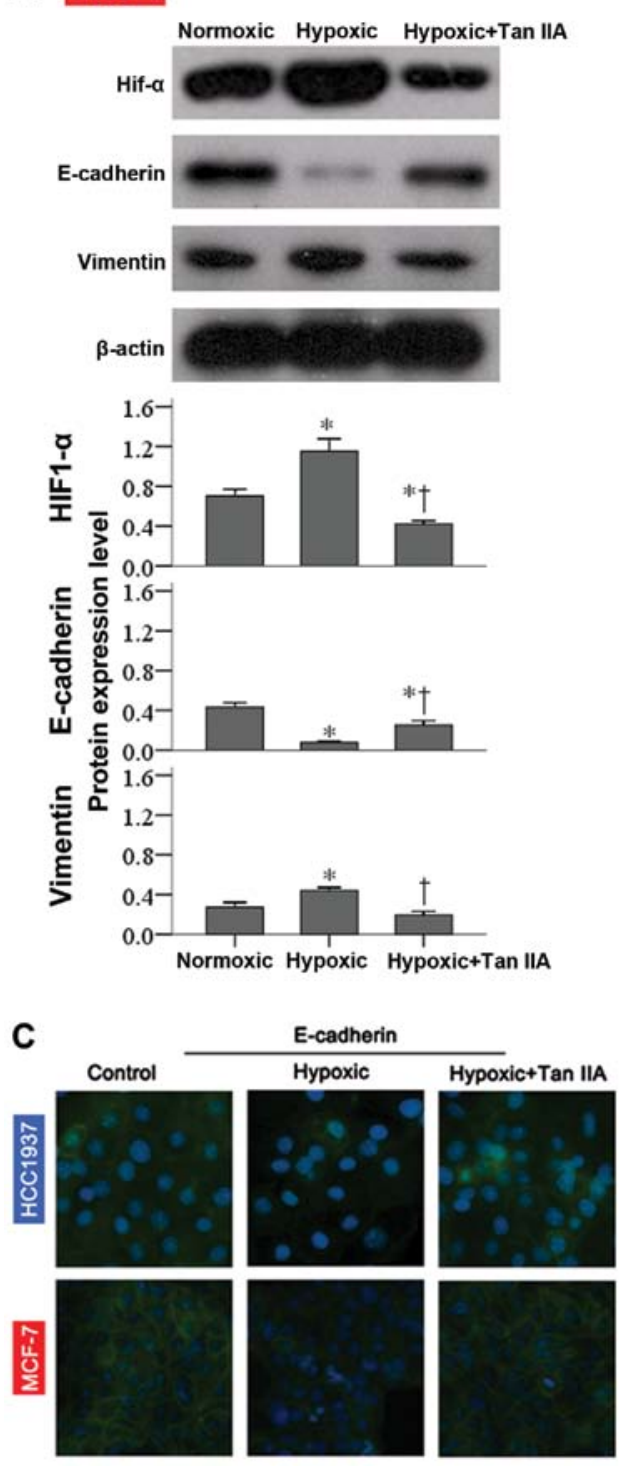

B $\mathrm{HCC1} 937$
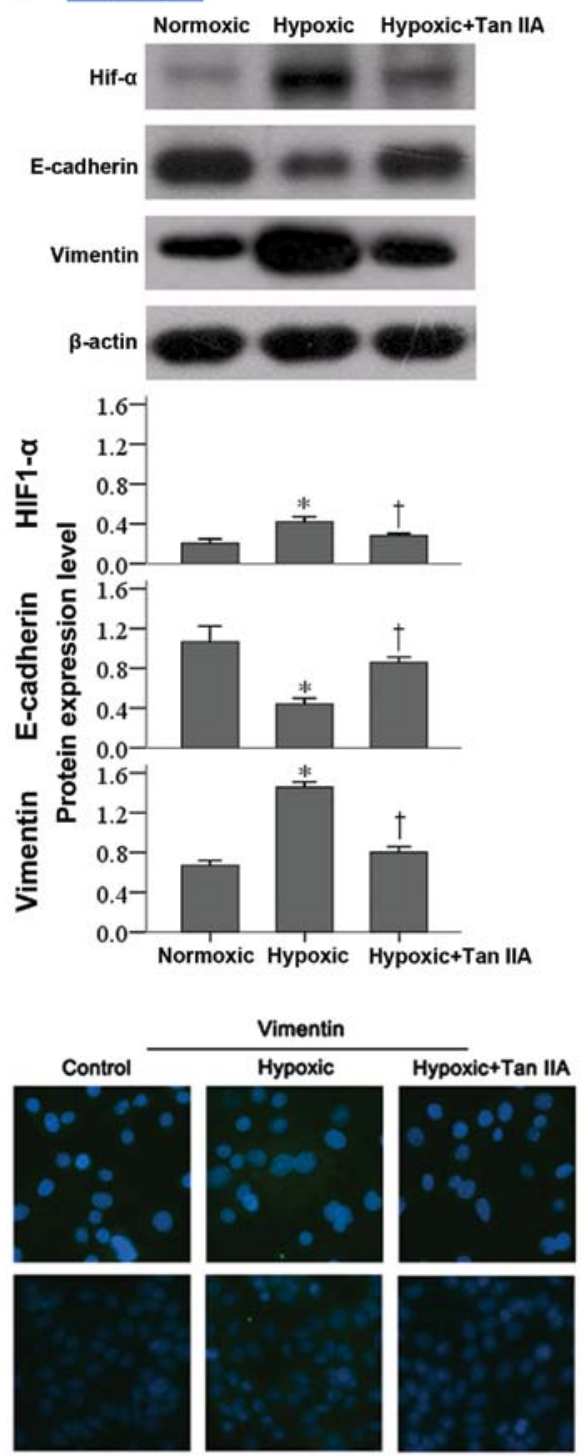

Figure 3. HIF-1 $\alpha$, E-cadherin and vimentin protein expression in response to hypoxia and Tan IIA. (A) MCF-7 and (B) HCC1937 cells were cultured in normoxia, hypoxia, and hypoxia+Tan IIA. (A and B) Western blot analysis of HIF-1 $\alpha$, E-cadherin, and vimentin protein expression was determined. (C) Immunofluorescence staining of MCF-7 and HCC1937 cells for E-cadherin and vimentin expression. ${ }^{*} \mathrm{P}<0.05$ compared to the normoxic group; ${ }^{\circ} \mathrm{P}<0.05$ compared to the hypoxic group.

A
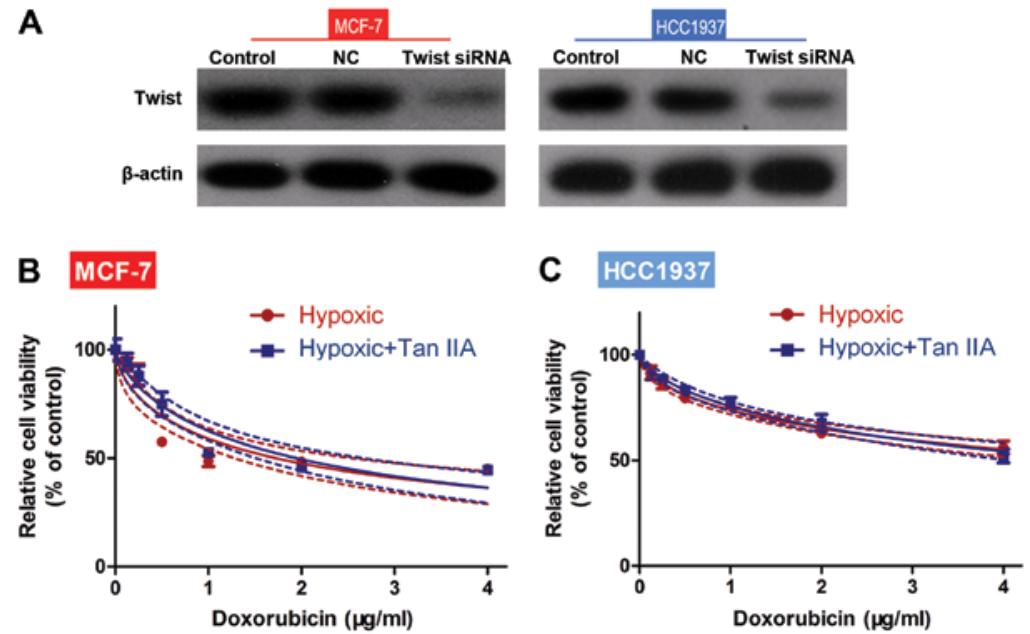

Figure 4. The effects Tan IIA are mediated by TWIST expression. (A) Western blot analysis of TWIST protein expression and (B and C) tumor cell viability after transfection with TWIST siRNA in the presence of hypoxia with and without Tan IIA. 
A
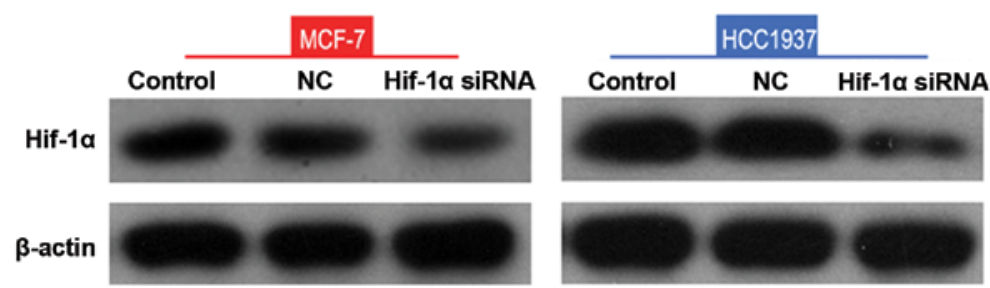

\section{B $\mathrm{MCF}-7$}

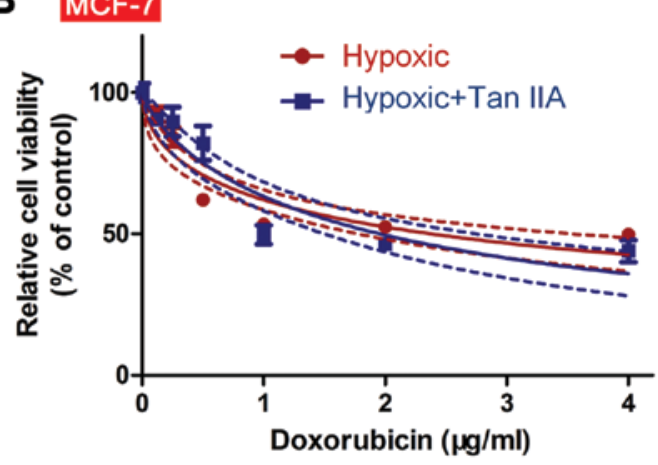

\section{C $\mathrm{HCC1937}$}

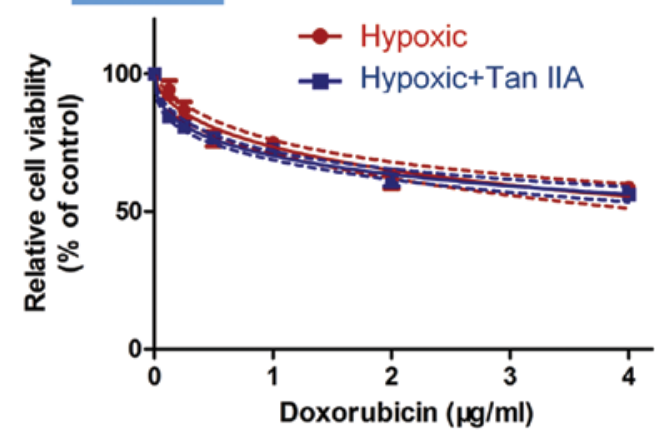

E $\mathrm{HCC} 1937$

D $\mathrm{MCF}-7$
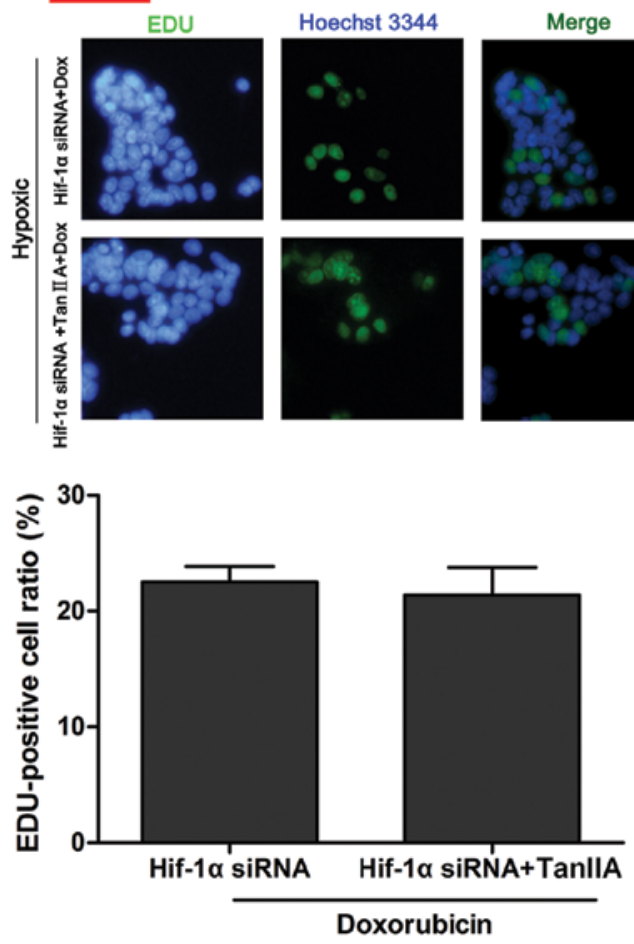

Merge
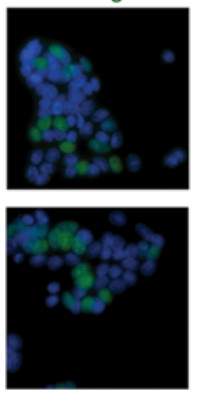

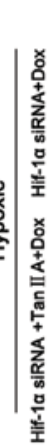
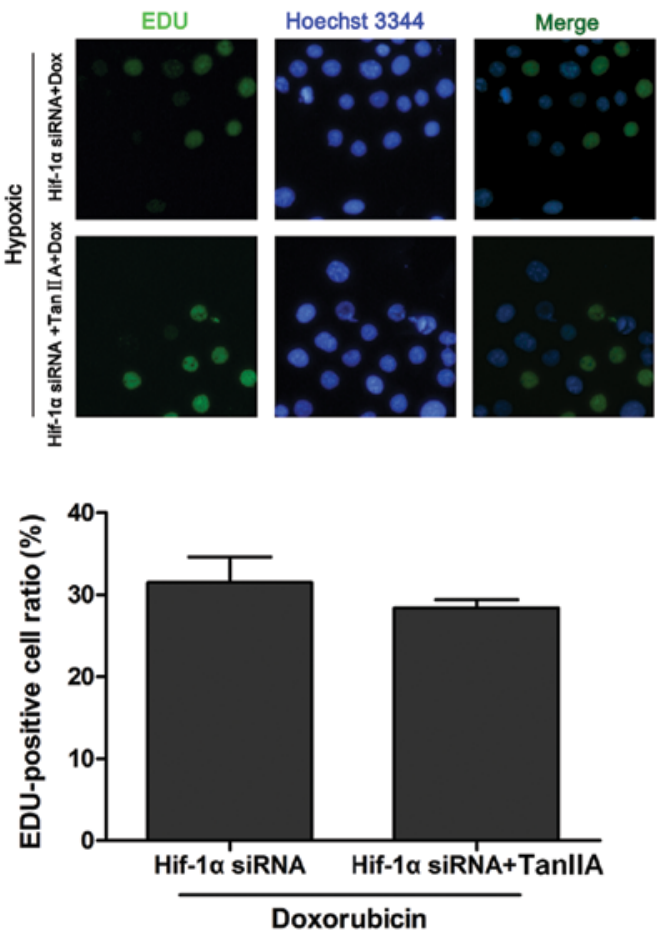

Figure 5. The effects Tan IIA are mediated by HIF-1 $\alpha$ expression. (A) Western blot analysis of HIF-1 $\alpha$ protein expression; (B and C) tumor cell viability, and (D and E) cell proliferation after transfection with HIF-1 $\alpha$ siRNA in the presence of hypoxia with and without Tan IIA.

\section{Discussion}

Considering the in vitro and in vivo growth inhibitory effects of Tan IIA on leukemia cells (15), prostate cancer cells (16), colon cancer cells (17), pancreatic cancer cells (18), hepatocellular carcinoma (19), gastric cancer cells (20), cervical cancer cells (21), and breast cancer cells (22), the effects of Tan IIA on hypoxia-induced DOX resistance were analyzed in two breast cancer cell lines. Tan IIA increased the sensitivity of both MCF-1 and HCC1937 cells cultured in hypoxia to DOX in part through HIF-1 $\alpha$. Tan IIA also reduced the expression of EMT markers, suggesting that it may play a role in reducing metastasis.

In the present study, Tan IIA reduced MCF-1 and HCC1937 cell viability and proliferation, suggesting that Tan IIA targets the cell cycle. Cell cycle arrest at the G0/G1 phase in response to Tan IIA has previously been reported in $\mathrm{LNCaP}$ prostate cancer cells (16) through p53 activation (23). Similar cell cycle arrest was observed in pancreatic (18), gastric (20) and cervical (21) cancer cells. However, Chiu et al (16) reported that these 
effects were mediated through endoplasmic reticulum (ER) stress. Further studies will assess the effects of Tan IIA on the cell cycle progression of both MCF-1 and HCC1937 cells.

Induction of apoptosis by Tan IIA in human leukemia cell lines through caspase-3 activation, downregulation of bcl-2 and bcl-xl and upregulation of bax has been reported (15). Similar results were reported for H146 small cell lung cancer cells (24), hepatocellular carcinoma (19), chronic myeloid leukemia cells (25) as well as BxPC-3 pancreatic cancer cells (18). However, no changes in apoptosis were observed in the present study. These differences may be due to cell-type specific effects of Tan IIA. Alternatively, Tan IIA may only induce apoptosis in normoxic conditions.

In the present study, Tan IIA increased the sensitivity of breast cancer cell lines to DOX, which is similar to that reported for SGC7901 gastric cancer cells in response to adriamycin and 5-fluorouracil (20). The mechanisms of cell growth inhibition by Tan IIA were also explored in vitro. The increased chemosensitivity observed with Tan IIA in hypoxia was in part mediated through HIF-1 $\alpha$. This is consistent with $\mathrm{Xu}$ et al (26) who reported that Tan IIA reduced LPS-induced sepsis syndrome through targeting HIF-1 $\alpha$. However, in hepatocellular carcinoma cells, HIF-1 $\alpha$ levels were not altered with Tan IIA in hypoxic conditions in vitro (12).

Differences in oxygen levels, presence of immune cells, growth factor expression as well as EMT between the center and periphery of solid tumors may result in resistance of some of these tumors to chemotherapies (27). Given the role of EMT in cancer progression and metastasis, the effects of Tan IIA on EMT marker expression were assessed. Hypoxia altered the expression of E-cadherin and vimentin EMT markers, which returned to control levels with Tan IIA in vitro. These results suggest that Tan IIA may inhibit metastasis (28) possibly through inhibition of HIF-1 $\alpha /$ TWIST-induced EMT (14). These results are partially consistent with Wang et al (12) who reported reduced EMT with Tan IIA in an in vivo model of hepatocellular carcinoma. However, similar results were not observed in hypoxic conditions in vitro (12). These inconsistencies may be due to differences in establishing the hypoxic conditions; they may also indicate cell-type differences in response to Tan IIA.

The present study is limited in that the pathway mediating changes in HIF-1 $\alpha$ expression in response to Tan IIA treatment was not investigated. Tan IIA activated the c-Jun N-terminal protein kinase (JNK) pathway in chronic myeloid leukemia cells (25) as well as the IL-6/STAT3/NF- $\kappa$ B signaling pathways in breast cancer cells (22); therefore, these pathways will be assessed in breast cancer cells in future studies. In addition, although the effect of Tan IIA on EMT markers is suggestive of inhibition of migration, further analyses will specifically assess the effects of Tan IIA on cell migration in vitro and metastasis in vivo. Furthermore, the results were not confirmed using in vivo studies, which will be undertaken in further analyses.

In conclusion, Tan IIA ameliorated hypoxia-induced chemotherapy resistance to DOX and EMT in breast cancer cell lines, which may be attributed to the downregulation of HIF-1 $\alpha$ expression. Further in vivo studies are required to fully elucidate the therapeutic potential of Tan IIA in increasing the sensitivity of breast cancer cells to chemotherapy.

\section{Acknowledgements}

The authors thank Zheng Xiaoxiao and Cai Ying for their assistance with the experimental techniques and data processing. This study was supported by a grant from the Traditional Chinese Medicine Research Foundation of Zhejiang Province (2013ZA076).

\section{References}

1. Redig A and McAllister S: Breast cancer as a systemic disease: a view of metastasis. J of Intern Med 274: 113-126, 2013.

2. Brown JM: Tumor hypoxia in cancer therapy. Methods Enzymol 435: 297-321, 2007.

3. Ryan HE, Poloni M, McNulty W, et al: Hypoxia-inducible factor-1alpha is a positive factor in solid tumor growth. Cancer Res 60: 4010-4015, 2000.

4. Vaupel P: The role of hypoxia-induced factors in tumor progression. Oncologist 9 (Suppl 5): 10-17, 2004.

5. Czekay RP, Aertgeerts K, Curriden SA and Loskutoff DJ: Plasminogen activator inhibitor-1 detaches cells from extracellular matrices by inactivating integrins. J Cell Biol 160: 781-791, 2003.

6. Giordano FJ and Johnson RS: Angiogenesis: the role of the microenvironment in flipping the switch. Curr Opin Genet Dev 11: 35-40, 2001.

7. Maxwell PH, Pugh CW and Ratcliffe PJ: Activation of the HIF pathway in cancer. Curr Opin Genet Dev 11: 293-299, 2001.

8. Semenza GL: Hypoxia-inducible factor 1: control of oxygen homeostasis in health and disease. Pediatr Res 49: 614-617, 2001.

9. Harada H: How can we overcome tumor hypoxia in radiation therapy? J Radiat Res 52: 545-556, 2011.

10. Luo Y, Lan L, Jiang YG, et al: Epithelial-mesenchymal transition and migration of prostate cancer stem cells is driven by cancer-associated fibroblasts in an HIF-1 $\alpha / \beta$-catenin-dependent pathway. Mol Cells 36: 138-144, 2013.

11. Shang Q, Xu H and Huang L: Tanshinone IIA: A promising natural cardioprotective agent. Evid Based Complement Alternat Med 2012: 716459, 2012.

12. Wang WQ, Liu L, Sun HC, et al: Tanshinone IIA inhibits metastasis after palliative resection of hepatocellular carcinoma and prolongs survival in part via vascular normalization. J Hematol Oncol 5: 69, 2012.

13. Chen Z, Zhang D, Yue F, Zheng M, Kovacevic Z and Richardson DR: The iron chelators Dp44mT and DFO inhibit TGF- $\beta$-induced epithelial-mesenchymal transition via up-regulation of N-Myc downstream-regulated gene 1 (NDRG1). J Biol Chem 287: 17016-17028, 2012.

14. Yang MH, Wu MZ, Chiou SH, et al: Direct regulation of TWIST by HIF-1alpha promotes metastasis. Nat Cell Biol 10: 295-305, 2008.

15. Liu JJ, Lin DJ, Liu PQ, Huang M, Li XD and Huang RW: Induction of apoptosis and inhibition of cell adhesive and invasive effects by tanshinone IIA in acute promyelocytic leukemia cells in vitro. J Biomed Sci 13: 813-823, 2006.

16. Chiu SC, Huang SY, Chen SP, Su CC, Chiu TL and Pang CY: Tanshinone IIA inhibits human prostate cancer cells growth by induction of endoplasmic reticulum stress in vitro and in vivo. Prostate Cancer Prostatic Dis 16: 315-322, 2013.

17. Su CC, Chen GW and Lin JG: Growth inhibition and apoptosis induction by tanshinone I in human colon cancer Colo 205 cells. Int J Mol Med 22: 613-618, 2008.

18. Huang CY, Chiu TL, Kuo SJ, Chien SY, Chen DR and Su CC: Tanshinone IIA inhibits the growth of pancreatic cancer BxPC-3 cells by decreasing protein expression of TCTP, MCL-1 and Bcl-xL. Mol Med Rep 7: 1045-1049, 2013.

19. Yuan SL, Wei YQ, Wang XJ, Xiao F, Li SF and Zhang J: Growth inhibition and apoptosis induction of tanshinone II-A on human hepatocellular carcinoma cells. World J Gastroenterol 10: 2024-2028, 2004.

20. Xu M, Cao FL, Li NY, Liu YQ, Li YP and Lv CL: Tanshinone IIA reverses the malignant phenotype of SGC7901 gastric cancer cells. Asian Pac J Cancer Prev 14: 173-177, 2013.

21. Pan TL, Hung YC, Wang PW, et al: Functional proteomic and structural insights into molecular targets related to the growth inhibitory effect of tanshinone IIA on HeLa cells. Proteomics 10: 914-929, 2010. 
22. Lin $\mathrm{C}$, Wang L, Wang $\mathrm{H}$, Yang L, Guo $\mathrm{H}$ and Wang $\mathrm{X}$ Tanshinone IIA inhibits breast cancer stem cells growth in vitro and in vivo through attenuation of IL-6/STAT3/NF- $\kappa$ B signaling pathways. J Cell Biochem 114: 2061-2070, 2013.

23. Won SH, Lee HJ, Jeong SJ, Lü J and Kim SH: Activation of p53 signaling and inhibition of androgen receptor mediate tanshinone IIA induced G1 arrest in $\mathrm{LNCaP}$ prostate cancer cells. Phytother Res 26: 669-674, 2012.

24. Cheng CY and Su CC: Tanshinone IIA may inhibit the growth of small cell lung cancer $\mathrm{H} 146$ cells by up-regulating the Bax/Bcl-2 ratio and decreasing mitochondrial membrane potential. Mol Med Rep 3: 645-650, 2010.

25. Yun SM, Jeong SJ, Kim JH, et al: Activation of c-Jun N-terminal kinase mediates tanshinone IIA-induced apoptosis in KBM-5 chronic myeloid leukemia cells. Biol Pharm Bull 36: 208-214, 2013.
26. Xu M, Cao F, Liu L, et al: Tanshinone IIA-induced attenuation of lung injury in endotoxemic mice is associated with reduction of hypoxia-inducible factor $1 \alpha$ expression. Am J Respir Cell Mol Biol 45: 1028-1035, 2011.

27. Nguyen L, Fifis T, Malcontenti-Wilson C, et al: Spatial morphological and molecular differences within solid tumors may contribute to the failure of vascular disruptive agent treatments. BMC Cancer 12: 522, 2012

28. Hu Y, Wang $\mathrm{S}, \mathrm{Wu} \mathrm{X}$, et al: Chinese herbal medicine-derived compounds for cancer therapy: a focus on hepatocellular carcinoma. J Ethnopharmacol 149: 601-612, 2013. 